\section{Drosophila let-7 microRNA is required for remodeling of the neuromusculature during metamorphosis}

\author{
Nicholas S. Sokol, ${ }^{1,3,6}$ Peizhang $\mathrm{Xu}^{2}$ \\ Yuh-Nung Jan, ${ }^{2}$ and Victor Ambros ${ }^{1,4,5}$ \\ ${ }^{1}$ Department of Genetics, Dartmouth Medical School, \\ Hanover, New Hampshire 03755, USA; ${ }^{2}$ Howard Hughes \\ Medical Institute, Department of Physiology, and Department \\ of Biochemistry and Biophysics, University of California at \\ San Francisco, San Francisco, California 94143, USA
}

The Drosophila let-7-Complex (let-7-C) is a polycistronic locus encoding three ancient microRNAs: let-7, miR-100, and fly lin-4 (miR-125). We find that the let-7-C locus is principally expressed in the pupal and adult neuromusculature. let-7- $C$ knockout flies appear normal externally but display defects in adult behaviors (e.g., flight, motility, and fertility) as well as clear juvenile features in their neuromusculature. We find that the function of let-7-C to ensure the appropriate remodeling of the abdominal neuromusculature during the larval-toadult transition is carried out predominantly by let-7 alone. This heterochronic role of let-7 is likely just one of the ways in which let-7-C promotes adult behavior.

Supplemental material is available at http://www.genesdev.org.

Received March 10, 2008; revised version accepted April 11, 2008.

Mutations in heterochronic genes in Caenorhabditis elegans cause cells in particular lineages to express their stage-specific fates earlier or later than normal (Ambros and Horvitz 1984). Detailed analysis of these genes has revealed a regulatory pathway of heterochronic genes that specifies the timing of cellular development in diverse cell types and thereby ensures a coordinated schedule of developmental events throughout the worm (for review, see Rougvie 2005; Moss 2007). The existence of the heterochronic gene pathway in worms and the conservation of some of its components through animal evolution suggest that functionally analogous pathways could also coordinate developmental timing in higher organisms (Pasquinelli et al. 2000). Two of these highly conserved components of the heterochronic pathway, let-7 and lin-4, are microRNAs (miRNAs), a class of small RNAs that post-transcriptionally modulate the ex-

[Keywords: let-7; let-7-Complex; microRNA; heterochronic; developmental timing; neuromuscular junction]

Present addresses: ${ }^{3}$ Indiana University, 1001 E. 3rd St., Jordan Hall A502, Bloomington, IN 47405, USA; ${ }^{4}$ University of Massachusetts Medical School, Program in Molecular Medicine, 373 Plantation St., Two Biotech, Suite 306, Worcester, MA 01605, USA.

Corresponding authors.

${ }^{5}$ E-MAIL vrambros@gmail.com; FAX (508) 856-5657.

${ }^{6}$ E-MAIL nsokol@indiana.edu; FAX (812) 855-6082.

Article is online at http://www.genesdev.org/cgi/doi/10.1101/gad.1671708. pression of target transcripts (for review, see Jackson and Standart 2007). The sequences and developmentally regulated expression profiles of let- 7 and lin-4 are conserved among diverse bilaterians (Pasquinelli et al. 2000; Sempere et al. 2003). For example, Drosophila let-7 and miR-125 (fly lin-4) are robustly up-regulated during metamorphosis, as is another highly conserved miRNA, miR-100 (Pasquinelli et al. 2000; Sempere et al. 2002, 2003; Bashirullah et al. 2003). All three of these ancient miRNAs are encoded in a $1-\mathrm{kb}$ region of the Drosophila genome (Fig. 1; Sempere et al. 2003), and their clustered organization has been conserved and duplicated in vertebrates (Supplemental Fig. S1; Sempere et al. 2003; Prochnik et al. 2007). These findings suggest that $m i R$ 100 , let-7, and miR-125 coordinately control gene expression to regulate developmental timing in animals. To test this hypothesis, we analyzed the roles of $m i R$ 100, let-7, and miR-125 in Drosophila and find that these miRNAs are required for normal adult behavior, suggesting roles in neural development and/or function. let-7 in particular is required for remodeling of the fly neuromusculature during the larval-to-adult transition, confirming that a general developmental timing function of let-7 has been evolutionarily conserved from worms to flies.

\section{Results and Discussion}

The clustered organization of Drosophila miR-100, let-7, and miR-125 suggests that these miRNAs are co-transcribed as a single polycistronic transcript. To test this hypothesis, we isolated cDNAs generated from genomic regions between $m i R-100$ and let-7 and between let-7 and $m i R-125$ using $5^{\prime}$ and $3^{\prime}$ rapid amplification of cDNA ends (RACE). This analysis identified two overlapping cDNA fragments that corresponded to a 2435nucleotide (nt) primary transcript that encoded the $\sim 70$ nt hairpin sequences of $m i R-100$, let-7, and $m i R-125$, and was comprised of three exons that spanned 17,400 kb of genomic DNA (Fig. 1A). We conclude that miR-100, let7, and $m i R-125$ are cotranscribed from a single locus, which we refer to as the let-7-Complex (1et-7-C) since let-7 was the first of these miRNAs identified in Drosophila (Pasquinelli et al. 2000). We infer that the miR100, let-7, and miR-125 clusters in the genomes of other animals (Supplemental Fig. S1) also represent single polycistronic loci. It should be noted that cotranscribed let-7-C miRNAs may not always be coexpressed, given that post-transcriptional processing of mature miRNAs from primary transcripts can be subject to developmental regulation (Thomson et al. 2006; Wulczyn et al. 2007; Viswanathan et al. 2008).

To investigate whether let-7-C miRNAs collectively regulate developmental timing in Drosophila, we generated two independent let-7-C knockout strains, let-7$C^{K O 1}$ and let-7-C $C^{G K I}$ (Fig. 1B,C; Supplemental Fig. S2). Both strains lack expression of the mature processed forms of $m i R-100$, let-7, and miR-125 (Fig. 1B,C). To reduce the potentially complicating effects of genetic background on our study, we analyzed the let-7- $C^{K O 1}$ and let-7-C $C^{G K I}$ knockout alleles in trans and refer to this trans-heterozygous let-7-C-null strain as let-7-C $C^{K O 1 / G K I}$. We found that $\sim 43 \%$ of $1 e t-7-C^{K O 1 / G K I}$ animals died prematurely during the course of development, with the majority $(74 \%)$ of these arresting at the very end of meta- 


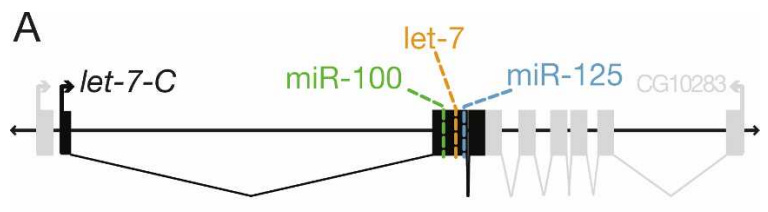

B

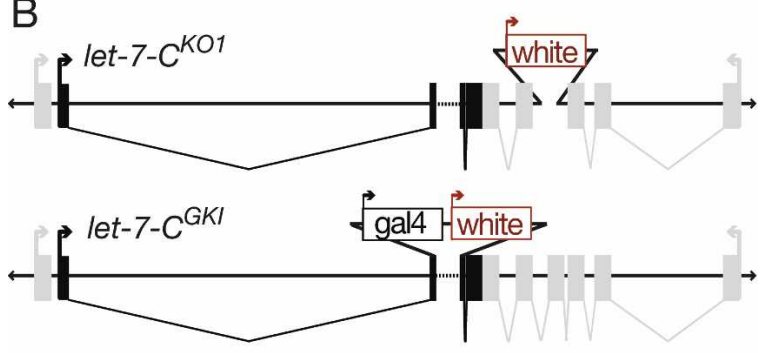

C

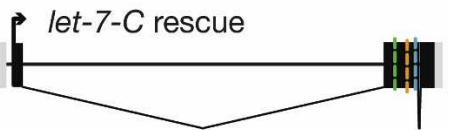

$\underline{2 \mathrm{~kb}}$

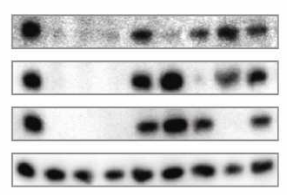

$\operatorname{miR}-100$

let-7

miR-125

miR-1

123456789

Figure 1. Drosophila let-7-C locus, knockouts, and rescuing transgenes. (A) The Drosophila let-7-C locus, located at cytological location 36E on chromosome 2, encodes a 2435-nt primary transcript containing three evolutionarily conserved miRNAs: miR-100, let-7, and miR-125. (B) The let-7-CKO1 and let-7-C $C^{G K I}$ mutations contain 1071- and 991-base-pair (bp) deletions respectively, removing $\mathrm{miR}$ 100, let-7, and $m i R-125$. The CG10283 gene, located proximally to let-7-C, is also disrupted by the let-7- $C^{K O 1}$ mutation. The let-7-C ${ }^{G K I}$ mutation contains Gal4 coding sequences driven by let-7-C transcription (see Supplemental Material for details on let-7- $C^{G K I}$ strain generation). $(C)$ The $P\{W 8$, let-7- $C\}$ rescuing transgene includes a 17,983-bp genomic fragment containing the let-7-C locus. Derivatives of $P\{1$ let-7- $C\}$ (not shown) contain 10- to 15-bp deletions removing portions of the mature miR-100, let-7, or miR-125 sequence (see the Supplemental Material for details of $P\left\{W 8\right.$, let-7- $\left.C^{\Delta m i r-100}\right\}$ $P\left\{W 8\right.$, let-7-C $\left.C^{\Delta l e t-7}\right\}$, and $P\left\{W 8\right.$, let-7- $\left.C^{\Delta m i R-125}\right\}$ transgene construction). (D) Expression of miR-100, let-7, and/or miR-125 RNA is eliminated in the let-7-C $C^{K O 1}$, let-7- $C^{G K I}$, and let-7-C $C^{K O 1 / G K I}$ strains and is restored by $P\{W 8,1 e t-7-C\}$ and derivative rescuing transgenes. Samples of total RNA from 1-d-old male flies of the following genotypes were analyzed by Northern blot: wild type in lane 1, let-7$C^{K O 1 / K O 1}$ in lane 2, let-7-C $C^{G K I / G K I}$ in lane 3, let-7-C $C^{K O 1 / G K I}$ in lane 4 let-7-C $C^{K O 1 / G K I} ; P\{W 8$, let-7-C $\}$ in lane 5, let-7- $C^{K O 1 / G K I}$; $P\{W 8$, let-7$\left.C^{\Delta m i r-100}\right\}$ in lane 6, let-7-C $C^{K O 1 / G K I} ; P\left\{W 8\right.$, let-7-C $\left.C^{\Delta l e t-7}\right\}$ in lane 7, let7-C $C^{K O 1 / G K I}$ i $P\left\{W 8\right.$, let-7- $\left.C^{\Delta m i R-125}\right\}$ in lane 8, and P\{UAS-let-7-C\}; let7-C $C^{K O 1 / G K I}$ in lane 9 . Northern blots were probed for miR-100, let-7, miR-125, and miR-1 RNAs, and miR-1 expression was used as a loading control.

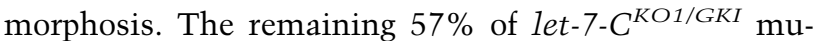
tants eclosed as adults, but displayed chronic defects in adult function, including severely reduced motility, flight, and fertility (Fig. 2). let-7-C KO1/GKI mutants that carried a transgene that restored let-7-C miRNA expression were fully rescued for developmental viability and adult functions (Fig. 2; data not shown). Despite their developmental and behavioral defects, let-7-C $C^{K O 1 / G K I}$ mutant pupae and adults appeared morphologically nor- mal (Supplemental Fig. S3), indicating that let-7-C miRNAs are not required for the morphogenesis of the adult exterior. These data indicated that let-7- $C$ expression is predominantly required for adult behavior and are consistent with the hypothesis that let-7-C miRNAs play an essential role in regulating the developmental remodeling of internal tissues during metamorphosis.

To test whether the activity of each of the let-7-C miRNAs is required for let-7-C function, we analyzed the phenotypes of three different let-7-C derivative strains in which the expression of $m i R-100$, let-7, or miR-125 had been eliminated individually (Fig. 1C; Supplemental Material). We refer to these singly mutant strains as $m i R-100^{\Delta}$, let- $7^{\Delta}$, and $m i R-125^{\Delta}$, respectively. miR-100 mutants functioned normally in all behavioral assays (Fig. 2), indicating that miR-100 was not solely responsible for any of the identified let-7-C functions. None of the single mutant strains displayed strong male fertility or climbing defects (Fig. 2D), suggesting that for normal male fertility and climbing behavior, the combinatorial action of any two let-7-C microRNAs could suffice. In contrast, $1 e t-7^{\Delta}$ and $m i R-125^{\Delta}$ mutants displayed severely reduced spontaneous locomotion as well as partial defects in flight (Fig. 2A-C). The normal climbing and nearly normal flight of $1 e t-7^{\Delta}$ and $m i R-125^{\Delta}$ mutants suggested that their severely impaired spontaneous locomotory activity was not simply the consequence of physically or metabolically impaired mobility, but rather likely reflected a behavioral deficit of neurological origin. Finally, let- $7^{\Delta}$ mutants alone displayed moderately severe defects in female fertility and oviposition (Fig. 2E,F), indicating that let-7 was required for an essential function to promote female reproduction.

To identify the specific place where let-7- $C$ miRNAs may function to promote adult behavior, we examined the spatiotemporal expression pattern of the 1et-7-C locus. We used the let-7-C $C^{G K I}$ strain, in which the yeast
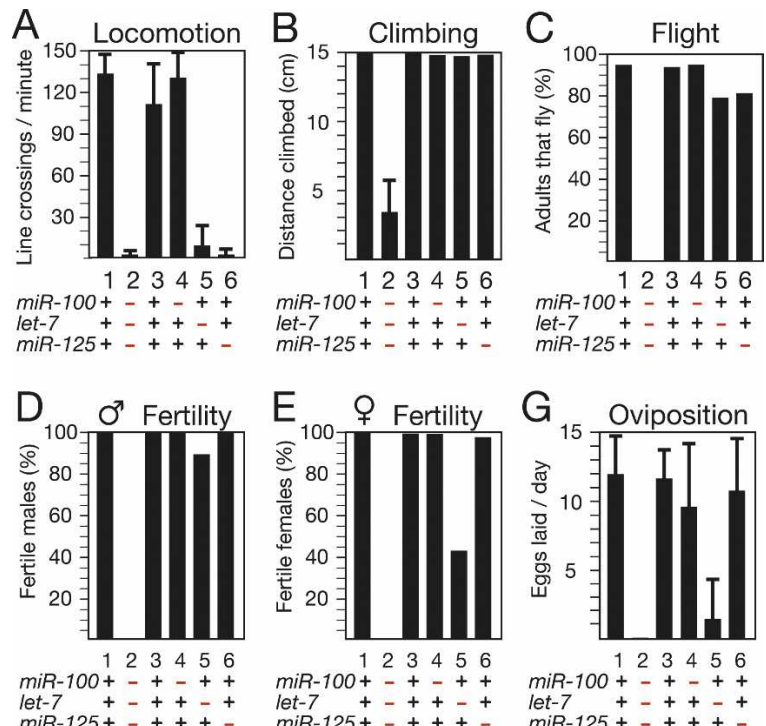

Figure 2. let-7- $C$ is required for normal adult behavior. For all assays, the following genotypes were analyzed: wild type in column 1 , let-7-C $C^{K O 1 / G K I}$ in column 2 , let-7-C $C^{K O 1 / G K I}$; $P\{W 8$, let-7-C $\}$ in column 3, let-7-C $C^{K O 1 / G K I} ; P\left\{W 8\right.$, let-7-C $\left.C^{\Delta m i r-100}\right\}$ in column 4 , let-7$C^{K O 1 / G K I} ; P\left\{W 8\right.$, let-7-C $\left.C^{\Delta l e t-7}\right\}$ in column 5 , and let-7- $C^{K O 1 / G K I} ; P\{W 8$, let-7-C $C^{\Delta m i R-125}$ in column 6 . For descriptions of the behavioral assays, see the Supplemental Material. 
transcriptional activator Gal4 had been inserted into the let-7-C locus (Fig. 1B), to drive expression of Gal4-dependent transgenes encoding membrane-bound or nuclear forms of GFP. A UAS-let-7-C transgene placed under the control of the let-7-C::Gal4 insertion restored miR-100, let-7, and miR-125 expression (Fig. 1D, lane 9) as well as climbing activity (Fig. 3A) to let-7-C $C^{K O 1 / G K I}$ mutants. Three characteristics of the 1et-7-C::Gal4 expression pattern are outlined below. First, let-7-C::Gal4 was expressed in neurons throughout the adult brain and ventral nerve cord, and this adult CNS expression was the culmination of a dramatic expansion in the spatial expression pattern of let-7-C::Gal4 that occurred in the CNS during the first half of metamorphosis (Fig. 3B; data not shown). Second, 1et-7-C::Gal4 was expressed in neurons that innervated structures throughout the adult (Fig. 3C), including sensory organs in the head, flight muscles in the thorax, and the alimentary tract, the male and female reproductive tracts, and the male and female genitalia in the abdomen (data not shown). We noted that let-7-C::Gal4 was very densely expressed in the posterior tip of the adult abdominal ganglion (Fig. 3B), as well as in motoneurons that projected posteriorly and innervated two distinct sets of abdominal muscles, the dorsal internal oblique muscles (DIOM) and the dorsal muscles (DM) (Fig. 3C). The DIOMs are remnants of the larval body wall that persist through metamorphosis (presumably to function in the process of eclosion) and in the wild type are fated to die within $12 \mathrm{~h}$ of eclosion (Crossley 1978; Kimura and Truman 1990). In contrast, the DMs are the adult body-wall muscles and are derived from larval myoblasts that undergo myogenesis during metamorphosis (Miller 1950; Currie and Bate 1991, 1995). Third, let-7-C::Gal4 was not only expressed in motoneurons but in muscle cells as well, including the DIOMs and DMs (Fig. 3E; data not shown). Taken together, the expression of let-7-C::Gal4 in pupal and adult neurons and muscles is consistent with the hypothesis that the behavioral phenotypes of let-7-C mutant adults are the consequence of defects in the metamorphosis of the neuromusculature.

To test whether let-7- $C$ miRNAs play a role in specifying the configuration of the adult neuromusculature, we examined the abdominal muscle system of let-7$C^{K O 1 / G K I}$ mutants since, as shown above, let-7-C is expressed in abdominal motoneurons and muscles. We found two very clear and highly penetrant defects (Fig. $4 \mathrm{~A}, \mathrm{~B})$. First, the DIOMs that ordinarily decay during post-eclosion maturation of wild-type flies failed to disappear in older let-7-C $C^{K O 1 / G K I}$ mutants (Fig. 4A,B). We quantified this phenotype by scoring the presence of six DIOMs in aged wild-type and let-7-C KO1/GKI mutant flies. Two-day-old wild-type males $(n=10)$ retained none of these DIOMs, whereas 2-d-old let-7-C $C^{K O 1 / G K I}$ males $(n=10)$ retained $89.9 \% \pm 11.7 \%$ of these DIOMs. Nineday-old male let-7-C $C^{K O 1 / G K I}$ mutants $(n=6)$, the oldest cohort of let-7-C $C^{K O 1 / G K I}$ mutants examined, retained $91.5 \% \pm 9.3 \%$ of these DIOMs. Second, the DMs of let7-C $C^{\text {KO1/GKI }}$ mutant adults were clearly smaller than those in age-matched wild-type controls (Fig. 4A-D). We quantified this phenotype by measuring the width and number of nuclei present in a set of approximately six to
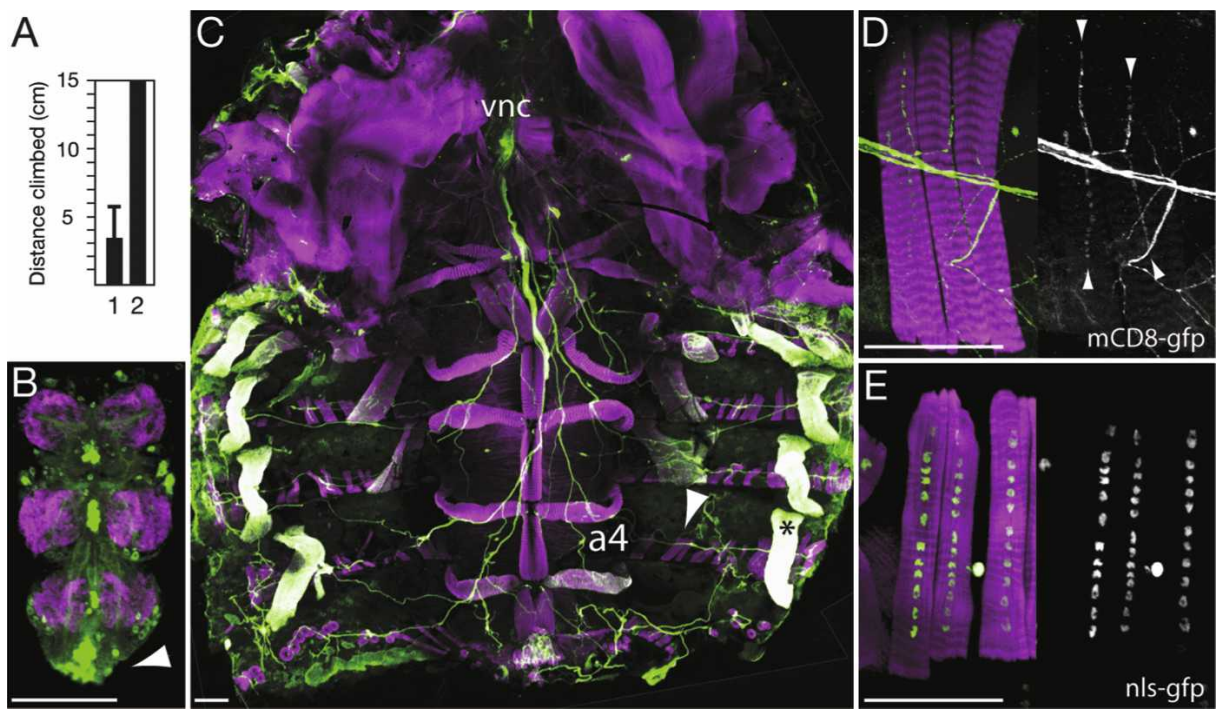

Figure 3. let-7-C is expressed in the nervous system and muscles of adult flies. (A) let-7- $C^{K O 1 / G K I}$ flies carrying a UAS-let-7-C transgene are rescued for climbing activity. (Column 1) let-7-C $C^{K O 1 / G K I}$; (column 2) let-7-C ${ }^{K O 1 / G K I} ; P\{U A S-1 e t-7-C\} /+$. $(B)$ Ventral section of the VNC of a let-7-C $C^{G K I} /+; P\{U A S-m C D 8-G F P\} /+$ fly stained for GFP (green) and the neuropil marker nc82 (purple). let-7-C::Gal4-driven GFP is expressed in neurons in the ventral nerve cord (VNC) and is enriched in the posterior tip of the abdominal ganglion (arrowheads). In all panels, anterior is up. (C) let-7-C:: Gal4 is expressed in neurons that project from the VNC posteriorly into the abdominal cavity. The carcass of a newly hatched let-7-C $C^{G K I} /+$; $P\{U A S-m C D 8$-GFP $\} /+$ male filleted on its dorsal midline and stained for GFP (green) and the F-actin marker rhodamine phalloidin (purple). GFP-positive neurons can be seen running along the nerve cord in each abdominal segment. In the fourth abdominal segment (A4), GFP+ neurons laterally contact the DMs (arrowhead) as well as the DIOMs (asterisk). The white appearance of the DIOMs indicates the colocalization of GFP and rhodamine phalloidin, signifying robust GFP expression. (D) let-7-C::Gal4 is expressed in motoneurons that innervate the DMs. DMs from a let-7-CGKI ++ P $\{U A S-m C D 8-G F P\} /+$ fly stained for GFP (green) and rhodamine phalloidin (purple). The GFP-only channel is also presented, and the neuromuscular junctions of $\mathrm{GFP}^{+}$neurons are indicated (arrowheads). (E) let-7-C:: Gal4 is expressed in the DMs. DMs from a let-7-C $C^{G K I}++;\{U A S-n l s-G F P\} / P\{U A S-n l s-G F P\}$ fly stained for GFP (green) and rhodamine phalloidin (purple). The GFP-only channel indicates expression of let-7-C $C^{G K I}$ in muscle cells. Expression of muscle cell GFP is also detectable in $D$, but is much less obvious. Bars: $A-C, 100 \mu \mathrm{m} ; D, E, 10 \mu \mathrm{m}$. 

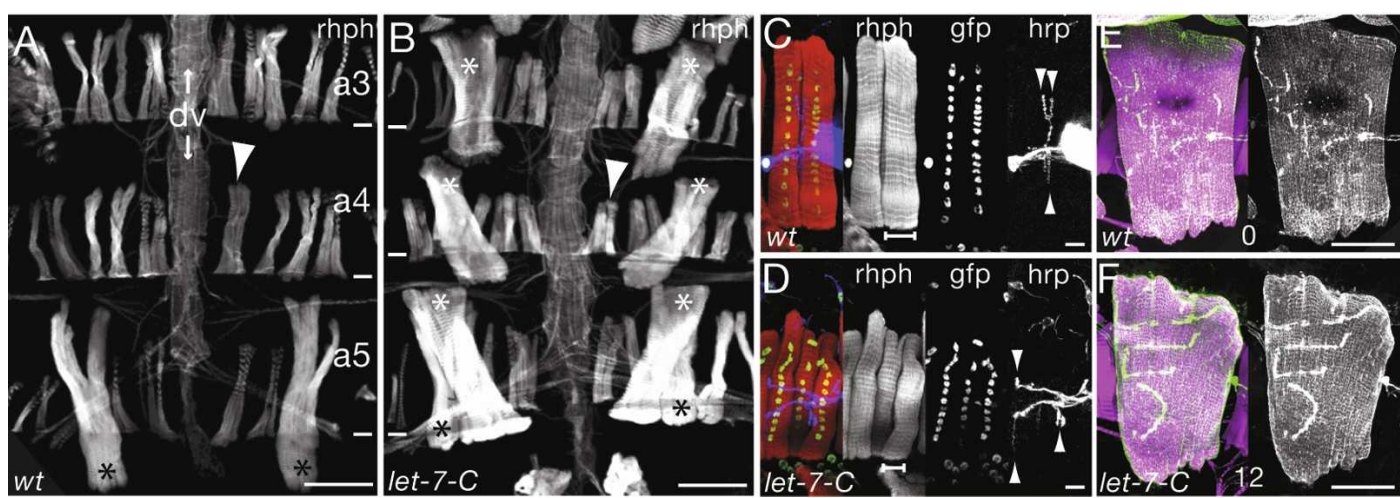

Figure 4. The adult neuromusculature of let-7-C $C^{K O 1 / G K I}$ mutants display persistent pupal as well as immature adult characteristics. $(A, B)$ Dorsal sections of abdominal segments 3-5 (A3-A5) from 2-d-old wild-type $(A)$ and let-7- $C^{K O 1 / G K I}$ mutant $(B)$ males stained for rhodamine phalloidin. The dorsal vessel (dv), which runs in an anterior-posterior orientation along the dorsal midline of the abdominal cavity, bisects the segments. $(B)$ In the let-7- $C^{K O 1 / G K I}$ mutant, a persistent DIOM is seen in each hemisegment (white asterisks). $(A, B)$ In addition, let-7-C ${ }^{K O 1 / G K I}$ mutant DMs are smaller than wild type (arrowheads). Male-specific muscles are apparent in A5 hemisegments in both wild type and mutant (black asterisks). $(C, D)$ Adult let-7-C $C^{K O 1 / G K I}$ mutant DMs and DM neuromuscular junctions (NMJs) do not grow to wild-type size. DMs from 2-d-old let-7-C $C^{G K I} /+; P\{U A S$ - nls-GFP $\} / P\{U A S-n l s-G F P\}(C)$ and let-7-C $C^{G K I} /$ let-7-C ${ }^{K O 1} ; P\{U A S-n l s-G F P\} / P\{U A S-n l s-G F P\}(D)$ males triple-labeled for rhodamine phalloidin (red), GFP (green), and HRP (blue). Rhodamine phalloidin-only, GFP-only, and HRP-only channels are also shown. 1et-7- $C^{K O 1 / G K I}$ mutant DMs are narrower (brackets), contain fewer nuclei, and are contacted by shorter NMJs (arrowheads). (E,F) let-7-C mutant DIOMs and innervating neurons persist into adulthood. DIOMs from 0-h-old let-7-C $C^{G K I} /+; P\{U A S-m C D 8-G F P\} /+(E)$ and $12-h-o l d$ let-7-C $C^{G K I} /$ let-7- $C^{K O 1}$; $P\{U A S-m C D 8-G F P\} /+(F)$ males double-labeled for rhodamine phalloidin (purple) and GFP (green). GFP-only channels are also shown. Bars: $A, B, 100 \mu \mathrm{m} ; C, D, 10 \mu \mathrm{m} ; E, F, 50 \mu \mathrm{m}$.

eight distinct DMs close to the dorsal vessel per A4 hemisegment per 2-d-old wild-type $(n=7)$ or let-7-C KO1/GKI $(n=7)$ male. Wild-type DMs were $20.2 \pm 2.7 \mu \mathrm{m}$ in width and contained $13.9 \pm 1.2$ nuclei, whereas let-7- $C^{K O 1 / G K I}$ DMs were $12 \pm 1.6 \mu \mathrm{m}$ in width and contained $10.6 \pm 0.8$ nuclei (Fig. 4C,D). Restoration of let-7-C expression rescued both the DIOM and DM phenotype; let-7-C $C^{K O 1 / G K I}$ mutants that carried the let-7- $C^{+}$transgene $(n=6)$ retained none of the six DIOMs scored above, and their DMs were $16.5 \pm 1.4 \mu \mathrm{m}$ in width and contained $12.8 \pm 0.6$ nuclei. To test whether let-7-C $C^{K O 1 / G K I}$ mutant muscle phenotypes were the consequence of defects apparent prior to the onset of metamorphosis, we examined the musculature and myoblasts of $1 e t-7-C^{K O 1 / G K I}$ larvae and found that both appeared normal (Supplemental Fig. S4). We therefore concluded that the abdominal muscle system of let-7- $C^{K O 1 / G K I}$ mutant adults failed to complete its larval-to-adult remodeling, displaying both persistent pupal as well as immature adult characteristics. We interpreted this as a heterochronic phenotype, since let-7-C $C^{K O 1 / G K I}$ mutant adults exhibited both juvenile features (e.g., muscle system morphology) as well as mature adult traits (e.g., external appearance) at the same time.

To test whether let-7-C affects the remodeling of other internal tissues, we examined the morphogenesis of the CNS during metamorphosis in let-7-C KO1/GKI mutants and found that at a gross level, CNS development appeared to have proceeded normally (data not shown). To examine the results of nervous system remodeling in finer detail, we focused on the morphology of motoneurons that innervate the DIOMs or the DMs. DIOMs are innervated by DIOM motoneurons, which also degenerate after eclosion. The DIOMs and their DIOM motoneurons, however, are triggered to die at different times and therefore may be controlled by independent signals (Kimura and Truman 1990). Interestingly, we found that the neuromuscular junctions (NMJs) connecting DIOMs and their innervating motoneurons failed to decay in let-
7- $C^{K O 1 / G K I}$ mutant adults (Fig. 4 E,F), indicating that the DIOMs and DIOM motoneurons persisted together. These data suggested that 1et-7-C functioned to coordinate the fates of DIOMs and DIOM motoneurons. Similarly, the reduced size of let-7-C $C^{K O 1 / G K I}$ mutant DMs was reflected in clear defects in let-7-C $C^{K O 1 / G K I}$ mutant DM NMJs, which were either completely absent, shorter in length than wild-type NMJs, or devoid of boutons, appearing as long, thin processes along the length of the DM (Fig. 4C,D). To quantify this phenotype, we measured the length of DM NMJs containing boutons in the same set of approximately six to eight DM muscles of wild-type $(n=7)$, let-7- $C^{K O 1 / G K I}(n=7)$, and rescued let7- $C^{K O 1 / G K I}(n=6)$ flies; wild-type NMJs were $55 \pm 7.8$ $\mu \mathrm{m}$ in length, let-7-C $C^{K O 1 / G K I}$ mutant NMJs were $11.2 \pm 1.6 \mu \mathrm{m}$ in length, and rescued let-7-C $C^{K O 1 / G K I} \mathrm{mu}-$ tant NMJs were $60.5 \pm 6.4 \mu \mathrm{m}$ in length. We concluded that the heterochronic abdominal muscle defect was reflected in a corollary nervous system defect, supporting the hypothesis that disruption of neuromusculature remodeling could underlie at least some of the let-7$C^{K O 1 / G K I}$ mutant behavioral phenotypes.

We note the striking similarity between let-7-C $C^{\text {KO1/GKI }}$ mutant phenotypes and the phenotypes associated with manual denervation of abdominal muscles prior to metamorphosis, reported by Currie and Bate in 1995. In both cases, adult DM muscles fail to grow to wild-type width, contain fewer nuclei, and display aberrant NMJs. However, the let-7-C mutation and denervation differ in at least one respect: their effect on the male-specific muscle of Lawrence (MOL) (Lawrence and Johnston 1986). MOLs are present in let-7-C $C^{K O 1 / G K I}$ adult males but absent in manually denervated adult males (Supplemental Fig. S5; Currie and Bate 1995). Interestingly, Currie and Bate did not report the persistence of DIOMs in denervated adults, which could mean either that DIOM degeneration is unaffected by denervation or that DIOMs degenerate precociously when denervated and were therefore not observed. In either case, the overall simi- 


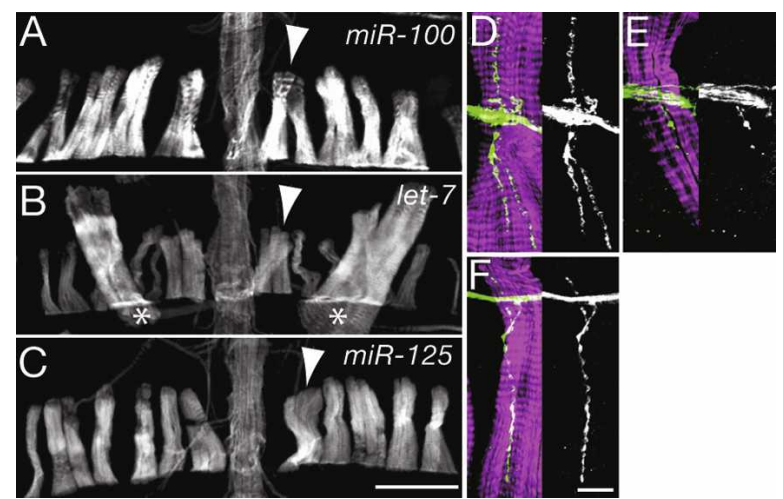

Figure 5. let-7 miRNA alone is required for the let-7-C-dependent larval-to-adult remodeling of the abdominal neuromusculature. (A$C)$ Dorsal sections of A4 segment from 2-d-old miR-100 $(A)$, let- $7^{\Delta}$ $(B)$, and $m i R-125^{\Delta}(C)$ males stained for rhodamine phalloidin. Note the persistent DIOMs (white asterisks) and small DMs (arrowheads) in let- $7^{\Delta}$ relative to $m i R-100^{\Delta}$ and $m i R-125^{\Delta} .(C, D)$ Adult let-7 DM neuromuscular junctions fail to achieve wild-type size. DMs from 2-d-old $m i R-100^{\Delta}(D)$, let-7 $7^{\Delta}(E)$, and $m i R-125^{\Delta}(F)$ males stained for rhodamine phalloidin (purple) and HRP (green). HRP-only channels are also shown. Bars: $C$ (applies to $A-C$ ), $100 \mu \mathrm{m} ; F$ (applies to $D-F$ ), $10 \mu \mathrm{m}$.

larity between the effects of genetic depletion of 1et-7-C and muscle denervation during metamorphosis supports the hypothesis that let-7-C is required to regulate an interaction between muscles and motoneurons during neuromusculature remodeling.

To test whether the activities of $m i R-100$, let-7, or $m i R-125$ are required individually for neuromusculature remodeling, we examined the abdominal muscle pattern as well as DM NMJs in $\mathrm{miR}-100^{\Delta}$, let- $7^{\Delta}$, and $\mathrm{miR}-125^{\Delta}$ single mutants (Fig. 5). We found that 2-d-old $m i R-100^{\Delta}$ $(n=6)$ and $m i R-125^{\Delta}(n=7)$ males retained none of the six DIOMs, while let- $7^{\Delta}$ males $(n=7)$ retained $61 \% \pm 28.5 \%$ of DIOMs. Although the frequency of complete DIOM retention is lower in $1 e t-7^{\Delta}$ mutants compared to let-7-C $C^{K O 1 / G K I}$ mutants, we noted that $83 \% \pm 25 \%$ of 1 let $-7^{\Delta}$ mutant DIOMs had arrested at some stage in the process of degeneration. With respect to both the DM and DM NMJ phenotype, we similarly found that $\operatorname{miR}-100^{\Delta}(n=5)$ and $\operatorname{miR}-125^{\Delta}(n=6) \mathrm{mu}-$ tants appeared normal, whereas let- $7^{\Delta}$ mutants $(n=6)$ phenocopied let-7-CKO1/GKI mutants. $m i R-100^{\Delta}$ and $\mathrm{miR}-125^{\Delta} \mathrm{DMs}$ were $18.3 \pm 0.8 \mu \mathrm{m}$ and $16.8 \pm 0.8 \mu \mathrm{m}$ in width, respectively, while let- $7^{\Delta}$ DMs were $12 \pm 1.8 \mu \mathrm{m}$ in length (Fig. 5A-C). Similarly, $m i R-100^{\Delta}$ and $m i R-125^{\Delta}$ NMJs were $45.9 \pm 10.2 \mu \mathrm{m}$ and $55.1 \pm 13.3 \mu \mathrm{m}$ in length, respectively, while let- $^{\Delta}$ NMJs were $12.5 \pm 5 \mu \mathrm{m}$ in length (Fig. 5D,E). For the sake of consistency, all the morphological data quantified in this study were collected from adult males. However, let-7-C KO1/GKI and let- $7^{\Delta}$ mutant females exhibited DM and DIOM phenotypes identical to their male siblings (data not shown), suggesting that the reduced egg-laying displayed by let$7^{\Delta}$ mutant females (Fig. 2F) might be a consequence of defects in their abdominal neuromusculature. From these data, we concluded that the activity of let-7 alone was predominantly responsible for let-7-C-dependent remodeling of the abdominal neuromusculature, and therefore that a heterochronic let-7 role in regulating developmental transitions had been evolutionarily conserved from worms to flies.
The functional dissection of Drosophila let-7- $C$ presented here indicates that let-7-C is required for adult behavior and that defects in neuromusculature remodeling correlate with some aspects of this requirement. We note that the perdurance of juvenile features in adult Drosophila let- 7 mutants is analogous to the reiteration of larval cell fates in adult Caenorhabditis elegans let-7 mutants (Reinhart et al. 2000), confirming the suggestion by Pasquinelli et al. in 2000 that let-7 might control developmental transitions in diverse bilateria (Pasquinelli et al. 2000). Future work in flies should extend this analysis to identify the relevant mRNA targets that Drosophila let-7 regulates in its heterochronic role and to examine how this heterochronic function is integrated into the more general requirements of the let-7-C locus in promoting adult behavior. For the most part, the set of targets predicted for Drosophila let-7 are distinct from those predicted for C. elegans let-7 (Grun et al. 2005; Lall et al. 2006). Our unpublished observations indicate that one of Drosophila let-7's targets is the transcription factor abrupt (Hu et al. 1995), although we also find that ectopic expression of abrupt in a let-7-C:: Gal4driven pattern is not sufficient to recapitulate the 1 et- $7^{\Delta}$ phenotype. The conservation of the genomic clustering as well as neuronal expression of 1et-7, mir-125, and mir100 from flies to vertebrates (Supplemental Fig. S1; Wienholds et al. 2005; Ason et al. 2006; Wulczyn et al. 2007) suggests that let-7-C loci could function in neuromuscular and/or neuronal remodeling in mammals. Future work on let-7-C should reveal how its diverse effects on temporal cell fates, developmental timing, and neuronal remodeling are related.

\section{Materials and methods}

\section{Drosophila strains and genetics}

Fly stocks were maintained at $25^{\circ} \mathrm{C}$ on standard media on a 12-h light, 12 -h dark cycle. Canton $S$ and/or $w^{1118}$ stocks were used as wild-type controls. Transgenic animals were generated using standard methods. Detailed descriptions of methods used to generate let-7-C mutant flies can be found in the Supplemental Material.

\section{Histochemistry}

Adult brains or abdomens were fixed in $4 \%$ paraformaldehyde for $1 \mathrm{~h}$ or $15 \mathrm{~min}$, respectively. Samples were washed in PBT, blocked for $1 \mathrm{~h}$ with $5 \%$ goat serum in PBT, and incubated overnight with primary antibodies, including rabbit anti-GFP (1:1000; Molecular Probes), mouse anti-nc82 (1:10; Developmental Studies Hybridoma Bank), mouse anti-22c10 (1:20; Developmental Studies Hybridoma Bank), rabbit anti-twist (1:4000; Siegfried Roth), and rabbit anti-HRP (1:500; Jackson Laboratories). Samples were washed in PBT and incubated with AlexaFluor 488 or 568 conjugated anti-mouse or anti-rabbit secondary antibodies (Invitrogen) and/or additional stains, including rhodamine phalloidin (1:1000; Sigma), Cy5 conjugated anti-HRP antibodies (1:200; Jackson Labs), and DAPI (1:10,000; Molecular Probes). Samples were washed and mounted in Vectashield (Vector Laboratories). Images were collected on a Leica confocal microscope.

The cDNA sequence of pri-let-7-C has been deposited in GenBank under accession number EU624487. Complete methods can be found in the Supplemental Material.

\section{Acknowledgments}

We thank the Developmental Studies Hybdridoma Bank, Yang Hong, and Jeff Sekelsky for reagents; members of the Ambros lab for illuminating discussions; and Yashi Ahmed and Claudio Pikielny for use of their fly room and injection facility. N.S.S. was supported by a postdoctoral fellowship from the Damon Runyon Cancer Research Fund (1728-02) and an equipment grant from the Hitchcock Foundation. P.X. was supported 
Sokol et al.

by a grant from the National Institute of Health (RO1 NS40929) to Y.N.J., who is an HHMI investigator. The Drosophila project in V.A.'s lab was supported by a National Institute of Health grant (GM066826).

\section{References}

Ambros, V. and Horvitz, R. 1984. Heterochronic mutants of the nematode Caenorhabditis elegans. Science 226: 409-416.

Ason, N., Darnell, D.K., Wittbrodt, B., Berezikov, E., Kloosterman, W.P., Wittbrodt, J., Antin, P.B., and Plasterk, R.H. 2006. Differences in vertebrate microRNA expression. Proc. Natl. Acad. Sci. 103: 1438514389.

Bashirullah, A., Pasquinelli, A.E., Kiger, A.A., Perrimon, N., Ruvkun, G. and Thummel, C.S. 2003. Coordinate regulation of small temporal RNAs at the onset of Drosophila metamorphosis. Dev. Biol. 259: 1-8.

Crossley, A.C. 1978. The morphology and development of the Drosophila muscular system. In The genetics and biology of Drosophila (eds. M. Ashburner and T.R.F. Wright), pp. 499-560. Academic Press, London.

Currie, D.A. and Bate, M. 1991. The development of adult abdominal muscles in Drosophila: Myoblasts express twist and are associated with nerves. Development 113: 91-102.

Currie, D.A. and Bate, M. 1995. Innervation is essential for the development and differentiation of a sex-specific adult muscle in Drosophila melanogaster. Development 121: 2549-2557.

Grun, D., Wang, Y.L., Langenberger, D., Gunsalus, K.C., and Rajewsky, N. 2005. microRNA target predictions across seven Drosophila species and comparison to mammalian targets. PLoS Comput. Biol. 1: e13. doi: 10.1371/journal.pcbi.0010013.

Hu, S., Fambrough, D., Atashi, J.R., Goodman, C.S., and Crews, S.T. 1995. The Drosophila abrupt gene encodes a BTB-zinc finger regulatory protein that controls the specificity of neuromuscular connections. Genes \& Dev. 23: 2936-2948.

Jackson, R.J. and Standart, N. 2007. How do microRNAs regulate gene expression? Sci. STKE 2007: re1. doi: 10.1126/stke.3672007re1.

Kimura, K.I. and Truman, J.W. 1990. Postmetamorphic cell death in the nervous and muscular systems of Drosophila melanogaster. J. Neurosci. 10: 403-411.

Lall, S., Grun, D., Krek, A., Chen, K., Wang, Y.L., Dewey, C.N., Sood, P., Colombo, T., Bray, N., Macmenamin, P., et al. 2006. A genome-wide map of conserved microRNA targets in C. elegans. Curr. Biol. 16: 460-471.

Lawrence, P.A. and Johnston, P. 1986. The muscle pattern of a segment of Drosophila may be determined by neurons and not by contributing myoblasts. Cell 45: 505-513.

Miller, A. 1950. The internal anatomy and histology of the imago of Drosophila melanogaster. In Biology of Drosophila (ed. M. Demerec), pp. 420-534. Wiley, New York.

Moss, E.G. 2007. Heterochronic genes and the nature of developmental time. Curr. Biol. 17: R425-R434.

Pasquinelli, A.E., Reinhart, B.J., Slack, F., Martindale, M.Q., Kuroda, M.I., Maller, B., Hayward, D.C., Ball, E.E., Degnan, B., Müller, P., et al. 2000. Conservation of the sequence and temporal expression of let-7 heterochronic regulatory RNA. Nature 408: 86-89.

Prochnik, S.E., Rokhsar, D.S., and Aboobaker, A.A. 2007. Evidence for a microRNA expansion in the bilaterian ancestor. Dev. Genes Evol. 2177: 3-7.

Reinhart, B.J., Slack, F.J., Basson, M., Pasquinelli, A.E., Bettinger, J.C. Rougvie, A.E., Horvitz, H.R., and Ruvkun, G. 2000. The 21-nucleotide let-7 RNA regulates developmental timing in Caenorhabditis elegans. Nature 403: 901-906.

Rougvie, A.E. 2005. Intrinsic and extrinsic regulators of developmental timing: From miRNAs to nutritional cues. Development 132: $3787-$ 3798.

Sempere, L.F., Dubrovsky, E.B., Dubrovskaya, V.A., Berger, E.M., and Ambros, V. 2002. The expression of the let-7 small regulatory RNA is controlled by ecdysone during metamorphosis in Drosophila melanogaster. Dev. Biol. 244: 170-179.

Sempere, L.F., Sokol, N.S., Dubrovsky, E.B., Berger, E.M., and Ambros, V. 2003. Temporal regulation of microRNA expression in Drosophila melanogaster mediated by hormonal signals and broad-Complex gene activity. Dev. Biol. 259: 9-18.

Thomson, J.M., Newman, M., Parker, J.S., Morin-Kensicki, E.M., Wright,
T., and Hammond, S.M. 2006. Extensive post-transcriptional regulation of microRNAs and its implications for cancer. Genes \& Dev. 16: 2202-2207.

Viswanathan, S.R., Daley, G.Q., and Gregory, R.I. 2008. Selective blockade of microRNA processing by Lin-28. Science 320: 97-100.

Wienholds, E., Kloosterman, W.P., Miska, E., Alvarez-Saavedra, E., Berezikov, E., de Bruijn, E., Horvitz, H.R., Kauppinen, S., and Plasterk, R.H. 2005. MicroRNA expression in zebrafish embryonic development. Science 309: 310-311.

Wulczyn, F.G., Smirnova, L., Rybak, A., Brandt, C., Kwidzinski, E., Ninnemann, O., Strehle, M., Seiler, A., Schumacher, S., and Nitsch, R. 2007. Post-transcriptional regulation of the let-7 microRNA during neural cell specification. FASEB T. 21: 415-426. 


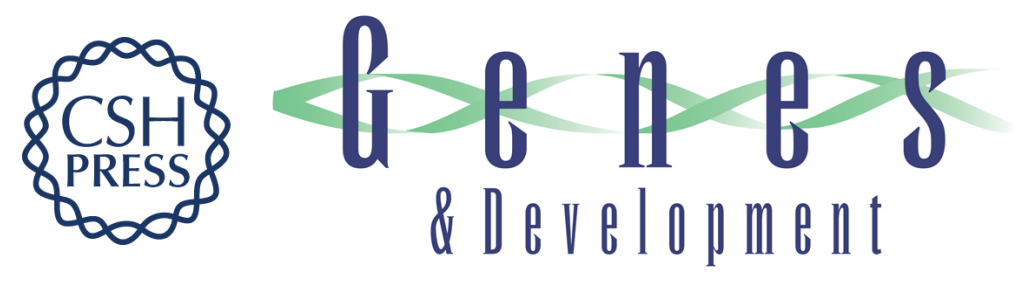

\section{Drosophila let-7 microRNA is required for remodeling of the neuromusculature during metamorphosis}

Nicholas S. Sokol, Peizhang Xu, Yuh-Nung Jan, et al.

Genes Dev. 2008, 22:

Access the most recent version at doi:10.1101/gad.1671708

\section{Supplemental http://genesdev.cshlp.org/content/suppl/2008/06/11/22.12.1591.DC1 \\ Material}

Related Content A matter of timing: microRNA-controlled temporal identities in worms and flies Manfred Frasch

Genes Dev. June , 2008 22: 1572-1576

References This article cites 22 articles, 9 of which can be accessed free at:

http://genesdev.cshlp.org/content/22/12/1591.full.html\#ref-list-1

Articles cited in:

http://genesdev.cshlp.org/content/22/12/1591.full.html\#related-urls

\section{License}

Email Alerting Receive free email alerts when new articles cite this article - sign up in the box at the top Service right corner of the article or click here.

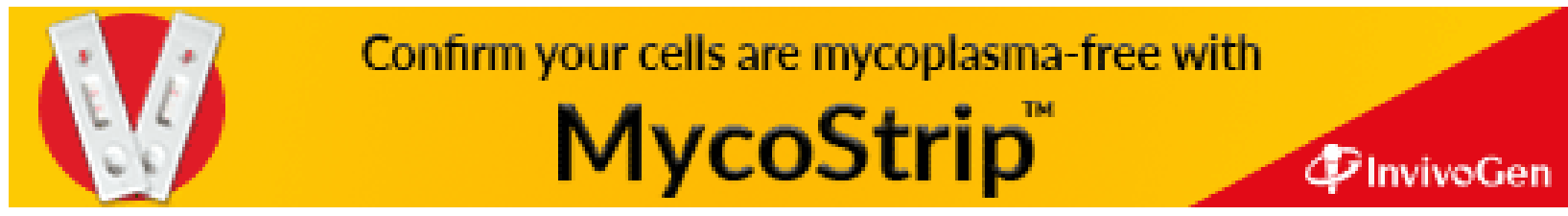

